\title{
EXCLUSÃO VASCULAR DO FÍGADO EM LESÕES VENOSAS HEPÁTICAS COMPLEXAS
}

\section{LIVER HEPATIC EXCLUSION IN COMPLEX HEPATIC VEIN INJURIES}

\author{
João Eduardo Leal Nicoluzzi, TCBC-PR' Luís Carlos VonBahten, TCBC-PR²; Gerson Luiz Laux, TCBC-PR³
}

\section{INTRODUÇÃO}

As lesões de vasos justa-hepáticos, veias suprahepáticas ou porção retro- hepática da veia cava, assim como da porção intra-hepática dos ramos venosos principais, são injúrias associadas a elevados índices de mortalidade. Os relatos isolados de pacientes que sobreviveram a este tipo de lesão foram na maioria manejado por técnicas de isolamento vascular do fígado, normalmente associado a shunt átriocaval. A alta mortalidade deste tipo de lesão deve-se a múltiplos fatores, incluindo a demora no diagnóstico, a falta de familiaridade com a técnica de shunt átrio-cava e uso do shunt quando as demais técnicas falharam ${ }^{1}$. O shunt átrio-cava apresenta mortalidade acima de $90 \%$ e outras manobras geralmente falham devido à hemorragia profusa associada à lesão de veias hepáticas ${ }^{2}$.

Atualmente as opções terapêuticas para o manejo destas lesões incluem: 1-manobra de Pringle, hepatotomia e sutura direta, 2- segmentectomia ou hepatectomia, 3- instalação de desvios átrio-cava ou femoro-axilar, 4- exclusão vascular do fígado (EVF), 5- transplante hepático.

Neste estudo apresentamos o relato de um caso de paciente com lesão de veia supra-hepática direita manejado por EVF.

\section{RELATO DO CASO}

Um paciente do sexo masculino, 27 anos, vítima de ferida por arma branca no hipocôndrio direito, foi recebido no Serviço de Emergência, sendo que ao exame inicial apresentava-se estável, com ferida única externa de $4 \mathrm{~cm}$, respirando espontaneamente e obedecendo ao comando verbal. Dada a presença de irritação peritoneal este paciente foi submetido à laparotomia exploradora.

Na cirurgia o paciente apresentava em torno de 300 $\mathrm{ml}$ de sangue livre na cavidade abdominal, sendo identificada lesão de 3 cm entre o segmento V e VI. À exploração da lesão com pinça de Kelly, constatou-se que a mesma prolongava-se profundamente no parênquima hepático. A exploração da ferida desencadeou sangramento profuso, o qual foi rapidamen- te controlado por compressão bi-manual do fígado. Como preparo para EVF foi feita reposição volumétrica tanto com cristalóides quanto com colóides, aumentando o nível da pressão venosa central acima do valor normal. Rapidamente foi dissecada a porção supra-hepática da veia cava através da liberação do ligamento falciforme até o plano dos vasos supra-hepáticos e lateralmente dos ligamentos triangulares até o plano da veia cava. Procedeu-se então à exclusão vascular do fígado com clampeamento seqüencial do pedículo hepático, da veia cava infra-hepática e da veia cava supra-hepática (Figura 1). A seguir fêz-se a hepatotomia por "keliclasia"onde foi diagnosticado lesão de veia supra-hepática direita, na sua porção intra-hepática, com extensão de mais ou menos $3 \mathrm{~cm}$. A lesão foi devidamente reparada com fio de polipropileno 4.0 cardiovascular. Após 25 minutos de isquemia os clampes foram liberados e realizou-se a hemostasia do parênquima

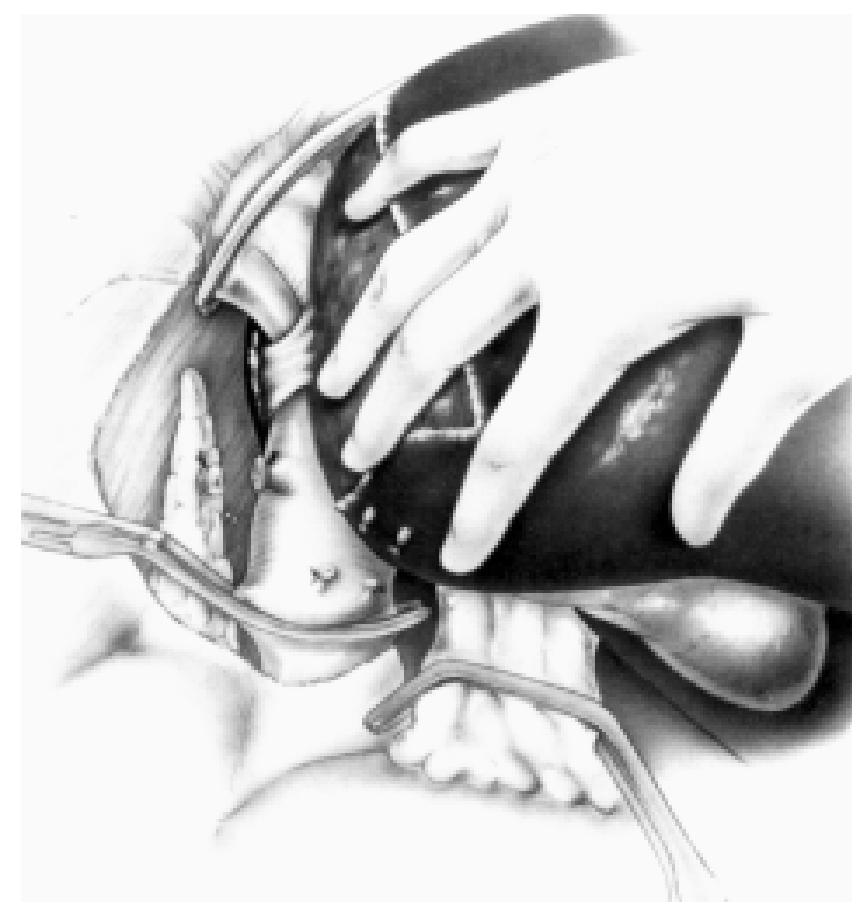

Figura 1 - Exclusão vascular do fígado com clampeamento do pedículo hepático, e da veia cava infra e supra-hepática.

1. Chefe do Serviço de Transplante Hepático e Pancreático do Hospital Angelina Caron; Mestre e Doutor em Cirurgia; Professor Adjunto da PósGraduação de Cirurgia da PUC-PR; Especialista em Cirurgia Hepato-Biliar, Hôpital Paul Brousse, Serviço Pr H. Bismuth, Paris, França; Cirurgião do Hospital Universitário Cajuru-PUC-PR.

2. Professor Titular de Cirurgia da PUC-PR; Mestre e Doutor em Cirurgia; Cirurgião do Hospital Universitário Cajuru-PUC-PR

3. Professor Adjunto de Cirurgia da PUC-PR; Preceptor da Residência de Cirurgia do Hospital Universitário Cajuru - PUC-PR.

Recebido em 05-01-06

Aceito para publicação em 04-03-05

Trabalho realizado no Serviço de Cirurgia Geral e do Trauma do Hospital Universitário Cajurú da PUC-PR, Curitiba PR. 
hepático por eletrocoagulação sem maiores problemas. $\mathrm{O}$ parênquima hepático foi deixado aberto e o abdômen foi drenado com dreno laminar de Penrose exteriorizado no flanco direito.

Na evolução este paciente apresentou fístula biliar de baixo débito por oito dias tendo recebido alta em perfeitas condições no $14^{\circ}$ dia de pós-operatório.

\section{DISCUSSÃO}

O tratamento do trauma hepático evoluiu muito nas últimas décadas. Inicialmente conduzido por laparotomia exploradora de rotina para todos os casos, o tratamento expectante atualmente é a regra no trauma fechado, reservando-se a laparotomia exploradora de rotina para a maioria dos casos de trauma aberto. Apesar destes avanços, nas lesões de vasos justa-hepáticos a exploração cirúrgica ainda é mandatória ${ }^{3}$.

O tratamento de lesões de vasos supra ou retro-hepáticos ainda é um grande desafio. A maioria das tentativas para acessar estes vasos estão associadas a grandes perdas hemorrágicas com alta mortalidade. O uso do shunt átrio-cava apresentaria o benefício teórico de diminuir a chance de hemorragia no acesso destes vasos, porém sua instalação é difícil e a maioria das séries apresentam resultados desapontadores com o seu uso ${ }^{3}$.

Recentemente alguns relatos publicados defendem nestas lesões a simples colocação de compressas (packing) com posterior reavaliação, ao contrário da idéia mais tradicional de ataque direto destas lesões ${ }^{4}$. Apesar do efeito da compressão ser útil na maioria dos casos, pode não ser suficiente em determinadas situações. O exemplo clássico é uma lesão de
Buckman tipo B, definida como uma lesão venosa extra hepática ${ }^{4}$. Neste tipo de lesão, pela ausência de tecidos de contensão ao seu redor, além da própria magnitude da hemorragia, pode ser necessária a aplicação de técnicas mais agressivas. Nestas situações poder-se-ia aplicar técnicas de clampeamento, como a EVF, usualmente empregadas no transplante hepático.

No caso ora apresentado, apesar de não ser uma lesão extra-hepática, não haveria motivo para contensão por compressas, uma vez que o paciente apresentava-se estável hemodinamicamente, além do que a compressão só retardaria o tratamento, aumentaria o índice de infecções e não descartaria a possibilidade de uma descompressão com sangramento abrupto. A EVF permitiu a correção da ferida de maneira exangue. Nas ocasiões em que o paciente apresente hipotensão acentuada pode-se tentar associar o clampeamento da aorta abdominal supra-celíaca ou, eventualmente, um shunt venoso femoro-axilar ${ }^{5}$.

Foi anteriormente descrito, em casos de clampeamento contínuo do parênquima hepático, tempos de isquemia de até 90 minutos em pacientes com fígado sadio e de no máximo 30 minutos em pacientes cirróticos. No caso de necessidade de tempos de isquemia mais prolongados, a maior experiência provém de cirurgias eletivas por ressecções tumorais de grande porte, através da perfusão de soluções refrigeradas através do parênquima hepático com conseqüente diminuição do metabolismo celular ${ }^{1}$.

Desta forma acreditamos que a EVF deve fazer parte das opções cirúrgicas do cirurgião do trauma, reservando-se seu uso para as graves lesões venosas tanto hepáticas quanto retro-hepáticas, sendo que a mesma ocuparia o lugar de diversas indicações da compressão por compressas de maneira mais efetiva.

\begin{abstract}
Injuries to the main hepatic veins carry a very high mortality rate, regardless of the technique used for repair. Isolated reports of survivors have, for the most part, been managed by hepatic vascular exclusion (EVE) techniques, usually with an atriocaval shunt. Herein we report a case of a severe intrahepatic major hepatic vein injury successfully managed with EVE (Rev. Col. Bras. Cir. 2006; 33(4): 258-259).
\end{abstract}

Key words: Liver/injuries; Hepatic veins/injuries.

\section{REFERÊNCIAS}

1. Pachter HL, Lianf HG, Hofstetter SR. Liver and biliary tract trauma. In: Feliciano DV, Moore EE, Mattox KL, editors. Trauma. $3^{\text {rd }}$ ed. Norwalk: Appleton \& Lange; 1996. p. 508-9.

2. Pachter HL, Spencer FC, Hofstetter SR, Liang HC, Coppa GF. The management of juxtahepatic venous injuries without an atriocaval shunts: preliminary clinical observations. Surgery. 1986;99(5):569-75.

3. Carrillo EH, Wohltmann C, Richardson JD, Polk HC Jr. Evolution in the treatment of complex blunt liver injuries. Curr Prob Surg. 2001;38(1):1-60.

4. Buckman RF Jr, Miraliakbari R, Badellino MM. Juxtahepatic venous injuries: a critical review of reported management strategies. J Trauma. 2000;48(5):978-84.
5. Malhotra AK, Fabian TC, Croce MA, Gavin TJ, Ki=udsk KA, Minard G, Pritchard FE. Blunt hepatic injury: a paradigm shift from operative to nonoperative management in the 1990s. Ann Surg. 2000;231(6):804-13.

Como citar este artigo:

Nicoluzzi JEL, Von Bahten LC, Laux GL. Exclusão vascular do fígado em lesões venosas hepáticas complexas. Rev Col Bras Cir. [periódico na Internet] 2006 Jul-Ago;33(4). Disponível em URL: http://www.scielo.br/rcbc

Endereço para correspondência:

Dr João Eduardo Leal Nicoluzzi

Rua: Santo Amaro, 118 - Água Verde

80620-330 - Curitiba - Paraná

Telefone: 41-2646719

E-mail: jenicoluzz@yahoo.com 